This item was submitted to Loughborough's Research Repository by the author.

Items in Figshare are protected by copyright, with all rights reserved, unless otherwise indicated.

\title{
Dynamic network function chain composition for mitigating network latency
}

PLEASE CITE THE PUBLISHED VERSION

https://doi.org/10.1109/ISCC.2018.8538646

PUBLISHER

(c) IEEE

VERSION

AM (Accepted Manuscript)

LICENCE

CC BY-NC-ND 4.0

REPOSITORY RECORD

Hajji, Wajdi, Thiago A. Genez, Fung Po Tso, Lin Cui, and lain W. Phillips. 2019. "Dynamic Network Function Chain Composition for Mitigating Network Latency". figshare. https://hdl.handle.net/2134/36912. 


\title{
Dynamic Network Function Chain Composition for Mitigating Network Latency
}

\author{
Wajdi Hajji*, Thiago Lopes Genez ${ }^{\dagger}$, Fung Po Tso*, Lin Cui ${ }^{\ddagger}$, Iain Phillips* \\ ${ }^{*}$ Department of Computer Science, Loughborough University, UK \\ ${ }^{\dagger}$ Institute of Computer Science, University of Bern, Switzerland \\ ${ }^{\ddagger}$ Department of Computer Science, Jinan University, Guangzhou, China \\ Email: w.hajji@lboro.ac.uk; genez@inf.unibe.ch; p.tso@lboro.ac.uk; tcuilin@jnu.edu.cn; i.w.phillips@lboro.ac.uk
}

\begin{abstract}
Network Function Virtualisation (NFV) enables rapid deployment of new services in networks on an on-demand basis using general purpose servers. Multiple virtual network functions (VNFs) can be dynamically chained in an ordered sequence for the delivery of end-to-end services. Nevertheless, network latency caused by the sequential order of packet processing on every VNF can hurt the performance of latency-sensitive applications. To reduce such network latency, existing solutions only consider the maximum capacity of individual virtual network functions (VNFs) and do not take into account the fact that performance of VNFs, as with any software applications, is bottlenecked by either CPU or I/O peripheral capacity of the server they run on and their underneath implementation such as singleor multi-threaded.

By exploiting this knowledge, we can better determine the number of required VNF instances and distribute the network traffic among them for any given VNF chains. In this paper, we formulate the VNF Scaling and Traffic Distribution problem and prove that it is NP-hard. We then present the design and implementation of Natif, an efficient VNF-Aware VNF insTantIation and traFfic distribution scheme. Through our OpenStack-based testbed evaluations, we demonstrate that Natif can significantly improve the network latency by $188 \%$ on average as compared to other approaches. As a chain composition scheme, Natif can effectively work with any VNF chaining algorithms.
\end{abstract}

\section{INTRODUCTION}

Virtual Network Functions (VNFs) (e.g., firewalls, load balancers) are often deployed in chains in between the communicating hosts [1]. Being in a chain, VNFs can provoke a mutual interference as they can change the volume of the processed traffic [2], which can cause resource, such as network and CPU, bottlenecks in the chain. As a result, network latency starts to build up and degrade the application performance, directly hurting revenue. It is reported that every $100 \mathrm{~ms}$ of latency cost Amazon 1\% in sales [3].

Current studies tackle the latency problem through VNF placement optimisation [4], VNF chaining [5][6] and VNF parallelisation [7]. These methods improve latency by shortening end-to-end paths. However, they see VNFs as black boxes and neglect their internal packet processing characteristics. VNFs

Corresponding author: Dr. Fung Po Tso

This work has been partially supported in part by the UK Engineering and Physical Sciences Research Council (EPSRC) grants EP/P004407/2 and EP/P004024/1; the Chinese National Research Fund (NSFC) No. 61772235 and 61402200; the Fundamental Research Funds for the Central Universities (21617409). are software applications running inside virtual environment. While software performance is bounded by either I/O or CPU or both of them, we argue that VNFs are no exceptions.

To demonstrate this, we have evaluated the resources usage at three VNFs, pfSense Network Address Translator (NAT), Snort Intrusion Detection System (IDS), and Suricata IDS. Each one of them is deployed on a Virtual Machine (VM) with $1 \mathrm{Gbps}$ vNIC, 1vCPU, and 1GB RAM. Fig. 1a depicts a clearly distinct CPU usage and CPU interrupts activities at pfSense NAT and Snort IDS in spite of being allocated similar resources and handling the same network traffic. This is because pfSense NAT is insensitive to the packet payload since it only handles the packet header. At each packet arrival it calls its subroutine running in the user space which results in high CPU interrupts (interrupt-driven I/O), hence I/O-bound. We have found that the effective computation of pfSense NAT is only around $2 \%$ of the total CPU usage. In comparison, the Snort IDS, using community rules ${ }^{1}$, buffers and inspects the data carried out in the packet payloads, a CPU-intensive task, hence CPU-bound. Interestingly, our experiments also revealed that Suricata IDS, a multi-threaded application, efficiently uses the CPU resources while Snort IDS, a single-threaded implementation, only uses one core at a time.

In this paper, we propose Natif $^{2}$, a VN-ㅡware VNF ins TantIation and traFfic distribution scheme, as a way to reconcile the discrepancy of VNF requirements in the network chains. Natif considers the correlation between traffic and VNF category (CPU- vs. I/O-bound). For instance, when instantiating an I/O-bound VNF, Natif determines the required number of its instances based on the packet rate of the total incoming/entering flows (i.e., the throughput). Then, it implements a flow-based load balancer method that primarily considers the packet rate of each flow in the traffic distribution on the existing VNF instances. However, when dealing with CPU-bound VNF, both VNF instantiation and traffic distribution consider the bandwidth of the incoming flows (the amount of received data). Also, we argue that Natif can be seamlessly integrated into the VNF management approaches since it does not interfere with the VNF placement or chaining

\footnotetext{
${ }^{1}$ https://www.snort.org/downloads/\#rule-downloads

${ }^{2}$ Natif is a French word that means innate, original, or natural
} 


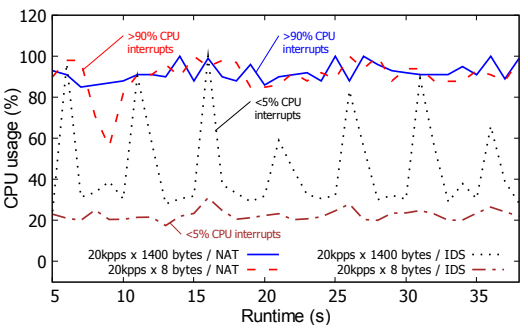

(a) CPU use at pfSense NAT and Snort IDS

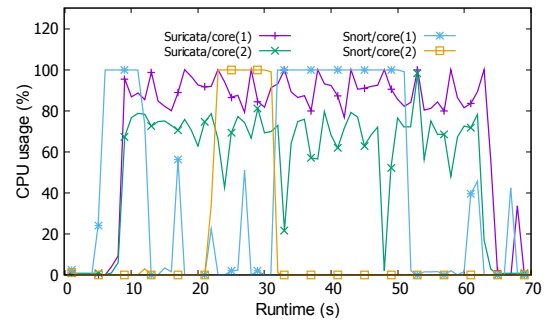

(b) Multi-threaded Suricata IDS vs. singlethreaded implementation in Snort

Fig. 1: VNF performance bottlenecks

but it complements them. In short, our contributions are fourfold:

- We experimentally demonstrate that an individual VNF's performance can be dependent on its host's CPU and/or I/O capacity (CPU- or I/O-bound), and their underpinning implementation method (single- vs. multi-threaded).

- We mathematically formulate the VNF scaling and traffic distribution problem and prove that it is NP-hard.

- We propose and implement Natif for efficiently compose given VNF chain based on VNFs' internal packet processing characteristics. We also open source our implementation $^{3}$ under the GNU General Public License.

- Our evaluation of Natif with OpenStack show that it can significantly improve the network latency by $188 \%$ on average as compared with other methods.

The remainder of this paper is structured as follows: Section II mathematically models the problem. Section III describes the proposed solution. Section IV presents the system design and implementation of Natif. Section V describes the experimental setup and evaluation. Section VI states the related work and Section VII concludes the paper.

\section{Problem Formulation AND Modelling}

We consider a network that can be modelled as a directed graph $G=(V, E)$ such as illustrated in Fig. 2, and we define the notations used in formulating the problem in Table I.

\footnotetext{
${ }^{3}$ https://github.com/SynNetSys/natif.git
}

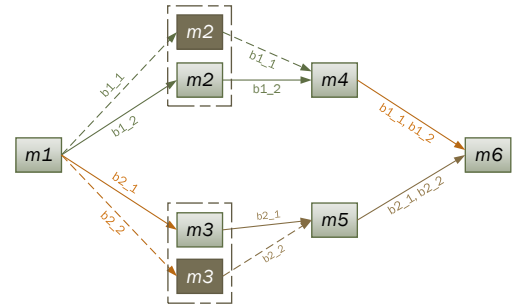

Fig. 2: Initially, this network chain had two branches, $b 1$ and $b 2$. After scaling out $m 2$ and $m 3, b 1$ and $b 2$ have been split into the sub-branches $\left(b 1_{1}, b 1_{2}\right)$ and $\left(b 2_{1}, b 2_{2}\right)$, respectively.

TABLE I: Notation

\begin{tabular}{|c|c|}
\hline Symbol & Description \\
\hline$M=\left\{m_{1}, m_{2}, \ldots\right\}$ & List of VNFs \\
\hline$G=(V, E)$ & $\begin{array}{l}G \text { directed graph. } V \subset M . E \text { links } \\
\text { between elements of } V\end{array}$ \\
\hline$C H=\left\{\operatorname{ch}_{1}, \operatorname{ch}_{2}, \ldots\right\}$ & List of network chains \\
\hline$B R_{i}$ & List of branches of $c h_{i} \in C H$ \\
\hline$S B_{i}$ & List of sub-branches of $c h_{i} \in C H$ \\
\hline$F=\left\{f_{1}, f_{2}, \ldots\right\}$ & List of flows \\
\hline$P=\left\{p_{1}, p_{2}, \ldots\right\}$ & List of policies \\
\hline$m_{i} . c, m_{i} \in M$ & Required CPU cores for $m_{i}$ \\
\hline$m_{i} . m$ & Required memory for $m_{i}$ \\
\hline$m_{i} . c a t$ & $\begin{array}{l}0 \text { if } m_{i} \text { is } \mathrm{I} / \mathrm{O} \text {-bound VNF, } 1 \text { if } \\
\text { CPU-bound }\end{array}$ \\
\hline$m_{i} \cdot c_{p p s}$ & $m_{i}$ capacity in packets per second \\
\hline$m_{i} \cdot c_{b p s}$ & $m_{i}$ capacity in bits per second \\
\hline$m_{i} . g d f$ & $\begin{array}{l}\text { Gain/drop factor of } m_{i} \text { : ratio of in- } \\
\text { coming to outgoing traffic travers- } \\
\text { ing } m_{i}\end{array}$ \\
\hline$m_{i} . g d_{p p s}$ & $\begin{array}{l}\text { Gain/drop factor in packets per sec- } \\
\text { ond }\end{array}$ \\
\hline$m_{i} . g d_{b p s}$ & Gain/drop factor in bits per second \\
\hline$m_{i} . t h d$ & $\begin{array}{l}0 \text { if } m_{i} \text { has single-threaded imple- } \\
\text { mentation, } 1 \text { if multi-threaded }\end{array}$ \\
\hline$f_{i} . s r c, f_{i} . d s t, f_{i} . b p s, f_{i} \cdot p p s$ & $\begin{array}{l}\text { Source, destination, bandwidth, } \\
\text { and packet rate, respectively, of } \\
f_{i} \in F\end{array}$ \\
\hline$p_{i}$. list,$p_{i}$. len & $\begin{array}{l}\text { List of deployed VNFs by } p_{i} \in P \\
\text { and its length, respectively. }\end{array}$ \\
\hline
\end{tabular}

The delay of flow when traversing a VNF is composed of a transmission delay and a processing delay. The former depends on the link capacity which is considered relatively stable in data centres [8]. The latter is due to the processing time, i.e. service time, of the incoming traffic plus the latency incurred by the packet queuing at the VNFs, i.e., the waiting time.

Let $D$ be the transmission delay matrix, where $D\left(m_{i}, m_{j}\right)=D\left(m_{j}, m_{i}\right)$ is the delay between $m_{i}$ and $m_{j}$, and $D\left(m_{i}, m_{j}\right)=-1$ if the delay is unknown or $m_{i}$ and $m_{j}$ are not reachable. We define the service time as the time that the VNF takes to process a packet or a bit of data based on its category. The service time of a VNF $m_{i}$ is given as follows.

$$
t_{s}^{i}=1 / m_{i} \cdot c_{p p s}
$$

For simplicity, we consider $\mathrm{M} / \mathrm{D} / 1$ queue at VNFs and VNFs process packets in a First-Come-First-Service (FCFS) discipline. We refer by $\lambda^{i}$ to the arrival rate of all flows traversing $m_{i} . \lambda^{i}$ is determined by a Poisson process and is defined as follows. 


$$
\lambda^{i}=\sum_{f_{i} \in E\left(*, m_{i}\right)} f_{i} \cdot p p s
$$

Given the utilisation $\rho_{i}=\lambda^{i} \times t_{s}^{i}$, the average waiting time $t_{w}^{i}$ of $m_{i}$ is

$$
t_{w}^{i}=\frac{t_{s}^{i} \times \rho_{i}}{2\left(1-\rho_{i}\right)}=\frac{\lambda_{i} \times t_{s}^{i^{2}}}{2\left(1-\lambda_{i} \times t_{s}^{i}\right)}
$$

And the processing delay of $m_{i}$ is:

$$
t_{p}\left(m_{i}\right)= \begin{cases}t_{s}^{i}, & \lambda_{i} \leq m_{i} \cdot c_{p p s} \\ t_{w}^{i}+t_{s}^{i}, & \text { otherwise }\end{cases}
$$

The packet rate (throughput) and bandwidth of the egress flow at VNF $m_{i}$ are defined as follow.

$$
\begin{aligned}
& f_{e} . p p s= \begin{cases}m_{i} \cdot g d_{p p s} \times \sum_{f_{i} \in E\left(*, m_{i}\right)} f_{i} \cdot p p s, & \text { if } \lambda_{i} \leq m_{i} \cdot c_{p p s} \\
m_{i} \cdot g d_{p p s} \times m_{i} \cdot c_{p p s}, & \text { otherwise }\end{cases} \\
& f_{e} . b p s= \begin{cases}m_{i} \cdot g d_{b p s} \times \sum_{f_{i} \in E\left(*, m_{i}\right)} f_{i} \cdot b p s, & \text { if } \lambda_{i} \leq m_{i} \cdot c_{p p s} \\
m_{i} \cdot g d_{b p s} \times m_{i} \cdot c_{d p s}, & \text { otherwise }\end{cases}
\end{aligned}
$$

where $f_{i} \in E\left(*, m_{i}\right)$ refers to all the flows entering $m_{i}$.

\section{A. VNF scaling and traffic distribution optimisation problem}

The expected delay of a flow $f_{i}$ traversing a branch $b_{i}$ governed by a policy $p_{i}$ is construed as follows.

$$
\begin{aligned}
& T\left(p_{i}\right)=D\left(f_{i} . s r c, p_{i} . \text { list }[1]\right) \\
& +\sum_{j=1}^{p_{i} . \text { len }-1}\left(D\left(p_{i} . \text { list }[j], p_{i} . \text { list }[j+1]\right)+t_{p}\left(p_{i} . \text { list }[j]\right)\right) \\
& +D\left(p_{i} . \text { list }\left[p_{i} . \text { len }\right], f_{i} . \text { dst }\right)
\end{aligned}
$$

Problem definition. Given the set of flows $F$, policies $P$, $V N F$ s $M$, chains $C H$, their branches $B R$, their sub-branches $S B$, and delay matrix $D$. We aim to determine the needed number of instances of each VNF to ensure that all ingress flows are accommodated. Afterwards, we map the flows on the resultant chain sub-branches based on both flow and VNF properties to minimise the total end-to-end delay at the chains.

$$
\text { Minimise } \sum_{p_{k} \in P} T\left(p_{k}\right) \quad \text { Subject to: }
$$

$\forall p_{k} \in P, p_{k}$ is satisfied

$\left\{\begin{array}{l}\forall p_{k} \in P, \forall m_{i} \in p_{k} . \text { list }, \\ \sum_{f_{i} \in E\left(*, m_{i}\right)} f_{i} \cdot \text { pps } \leq \sum_{m_{j} \in M_{i}} m_{j} . c_{p p s}, \quad m_{i} . \text { cat }=0 \\ \sum_{f_{i} \in E\left(*, m_{i}\right)} f_{i} . \text { bps } \leq \sum_{m_{j} \in M_{i}} m_{j} . c_{d p s}, \quad \text { otherwise }\end{array}\right.$

$\forall f_{i} \in E\left(*, m_{i}\right), \exists m_{j} \in M_{i}$,

$\left\{f_{i} \cdot\right.$ pps $\leq m_{j} . c_{p p s}, \quad m_{i} \cdot$ cat $=0$

$f_{i} . b p s \leq m_{j} \cdot c_{d p s}, \quad$ otherwise
The constraints (C1) impose that all policies should be satisfied. (C2) ensure that there should be $\left|M_{i}\right|$ VNFs capable of handling all the incoming flows, either for I/O-bound or CPU-bound VNFs. (C3) highlight that in a flow-based distribution scheme, we might have individual flows that cannot be accommodated by any of the VNF instances. Hence, we ensure that for any flow traversing a group of VNF instances, there should be an $m_{i}$ that has the sufficient capacity.

The above problem can be proven to be NP-Hard.

Proof. Consider a special case that includes a chain of three VNFs of $m_{1}, m_{2}$, and $m_{3}$ (in one branch) and is processing $n$ flows. We suppose that initially $m_{1}$ has not the sufficient resources to handle the $n$ flows but the other VNFs do. A reasonable solution is to scale out $m_{1}$ by at least one unit. In this new setup, the main branch will be split into two sub-branches. Then, the original problem becomes to find an appropriate sub-branch for each of the $n$ flows that results in the overall low latency. Consider each flow to be an item, where its requirement (throughput and bandwidth) is the item size. Thus, each VNF can be seen as a knapsack $k_{j}$ with limited capacity $k_{j}$.cap. The profit of assigning flows to each VNF is the negative of the flow delays. Then, our problem becomes finding a path for each flow through the VNFs that maximises the total profit. In other words, this becomes a Multiple Knapsack Problem (MKP) [9], whose decision version has already been proven to be NP-hard. Therefore, the MKP problem is reducible to our problem in polynomial time, and hence our problem is NP-hard.

\section{VNF INSTANTIATING AND TRAFFIC DISTRIBUTION}

In this section, we briefly describe the three principles of our proposed solution.

\section{A. VNF instantiation}

We calculate the total packet rates and bandwidth of all flows traversing each branch of the chain. Then, depending on the VNF category (I/O- or CPU-bound), we determine how many instances needed for that VNF. We calculate the new flow attributes after traversing a VNF $m_{i}$ based on the gain/drop factor. Afterwards, we run the VNF instantiation and we carefully allocate the number of cores to each instance $\left(m_{i} . c\right)$ by considering their internal implementation reflected by the variable $m_{i}$.thd (single- versus multi-threaded). In particular case, if the VNF is single-threaded then the number of allocated cores will be the number of needed instances.

\section{B. VNF-aware traffic distribution}

In the flow mapping against the instances of a VNF $m_{i}$, we aim to reduce the likelihood of having no accommodated flows. Therefore, we start assigning the flows with the highest bandwidth if $m_{i}$ is CPU-bound or the highest packet rate if it is $\mathrm{I} / \mathrm{O}-$ bound to the instances having the maximum remaining capacity (either in bits per second if $m_{i}$ is CPU-bound or packet per second if $m_{i}$ is I/O-bound). Then, the flow status (mapped or not) and the VNF remaining capacity will be 


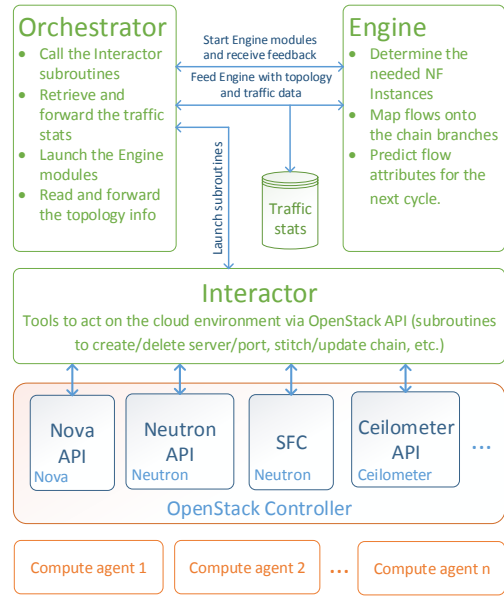

Fig. 3: System design

updated, and we iterate the process with the other VNF in the chain. For a given VNF, the mapping finishes when there is no flow left untreated.

However, solely relying on the VNF category and the flow attributes can lead to poor mapping decisions. For instance, if at a CPU-bound VNF, we are receiving flows with negligible bandwidth, relatively to the VNF capacity in bits per second, but with high packet rate, considering only the bandwidth in the traffic distribution may create a congestion at one of the VNF instances. So we propose that if the flow bandwidth is negligible comparing to the capacity in bits per second of a CPU-bound VNF, then we consider the flow rate instead. Also, if the flow rate is negligible relatively to an I/O-bound VNF capacity in packets per second, we then consider the flow bandwidth in the traffic distribution.

\section{Traffic characteristics prediction}

Our proposal requires the identification of flow attributes over a period of time (e.g., a cycle). Therefore, we use the traffic statistics logged at the forwarding devices (OVS switch) to train a prediction algorithm to help in characterising the coming flows. For this purpose, we use the prediction model Arima [10] to determine the flows attributes. Without the output of the prediction or for bursty traffic, any new flow entering the network chain will be mapped to the set of the VNF instances in respect to the associated policy and based on its current attributes, i.e., at the arrival time.

Our controller makes decisions after each cycle of the traffic prediction run. The quality and reliability of working data, as well as their rate of change, determine the period of the cycle, which is in the order of minutes in our implementation.

\section{SySTEM DESIGN AND IMPLEMENTATION}

\section{A. System architecture}

Natif is implemented in OpenStack ${ }^{4}$ environment. Its modules are deployed alongside the OpenStack controller. Our source code, around 1,500 lines, is written with OpenStack Python API. The controller has three main blocks. The Interactor provides tools to instantiate VNFs, create network chains

\footnotetext{
${ }^{4}$ https://www.openstack.org/software/newton/
}

and perform traffic steering. The Engine implements Natif's logic presented in section III. The Orchestrator synchronises between the different modules as shown in Fig. 3.

\section{B. Controller modules}

- Chain reading and creation module reads the configuration files describing the chains details and then instantiates the VNFs on VMs.

- Flow mapping determines the path of each flow and calls the Service Function Chaining (SFC) subroutines to implement the forwarding rules.

- Chains update module updates the flow mapping by implementing/deleting the forwarding rules after each change on the chain topology.

- Flow attributes prediction module collects statistics on the traffic traversing the chain to train the Arima prediction model and then determines to flow attributes for the next cycle.

\section{EXPERIMENTAL EVALUATION}

\section{A. Testbed experiment}

We deploy OpenStack (one controller, two compute, and one storage nodes) on two servers with four 1 Gbps NICs, 8 CPU cores and 32GB RAM each. The experiment involves chains of length three composed of NAT, firewall (FW) as representative of I/O-bound VNF and IDS for CPU-bound category. Measurements have been taken during five consecutive cycles of Arima prediction. We define three network traffic flavors/profiles. Profile 1 (P1): Six flows at high packet rate (20kpps) but low bandwidth (8-byte packet payload). Profile 2 (P2): Six flows with high bandwidth (1400-bytes payload) but random packet rates (normal distribution from $5 \mathrm{kpps}$ to 20kpps). Profile 3 (P3): mixed flows from P1 and P2. We also use two reference scenarios for the performance assessment. Greedy: it relies on the resources overprovisioning. It scales up all the VNFs by equally allocating all the available CPU cores and memory to them. Stratos-based ${ }^{5}$ (StratosB) [1]: It determines the number of instances of each VNF and distributes traffic based on the total bandwidth of the ingress flows. Thus, StratosB ensures a network-aware traffic distribution to reduce the likelihood of the network congestion and link saturation. For each scenario, we apply the three profiles, and then we measure the chain throughput (Mbps) and end-to-end delay.

\section{B. Network metrics assessment}

Fig. 4 shows how Natif has mitigated the RTT in the three profiles. Fig. 4 a illustrates how, at the $90^{\text {th }}$ percentile, Natif achieves $85 \%$ and $100 \%$ improvement compared to Greedy and StratosB, respectively. Fig. 4b shows the RTT for P2, StratosB outperforms Greedy, but it is still less efficient than Natif. When we apply P3 (Fig. 4c), Natif still outperforms Stratos $B$ as it has less RTT, $15 \mathrm{~ms}$ compared to $30 \mathrm{~ms}$ at $99^{\text {th }}$ percentile in StratosB, which means an improvement of nearly $100 \%$. It also achieves better results comparing to Greedy as

\footnotetext{
${ }^{5}$ Implemented on https://github.com/wajdihajji/natif.git
} 


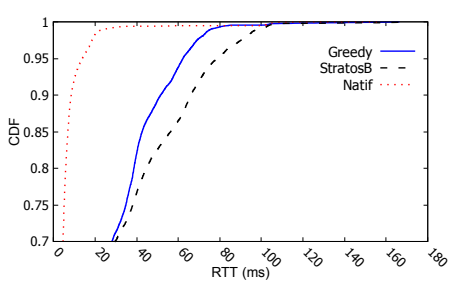

(a) Profile 1: high packet rate flows

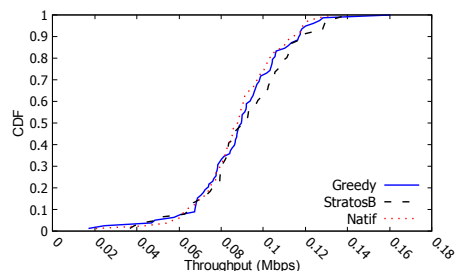

(a) Profile 1

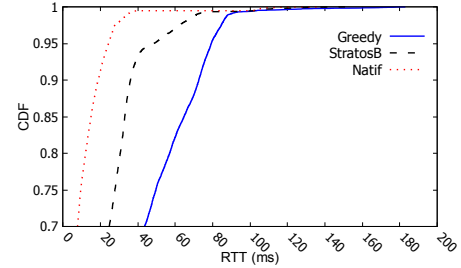

(b) Profile 2: high throughput flows

Fig. 4: RTT (ms)

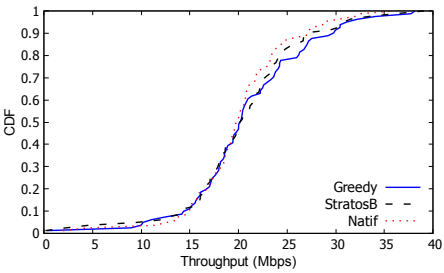

(b) Profile 2

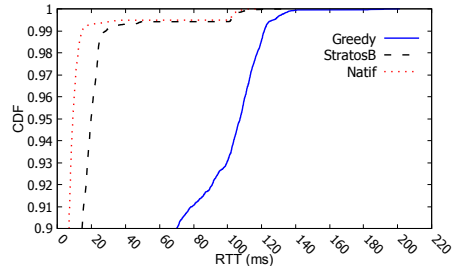

(c) Profile 3: Mix of profiles 1 and 2

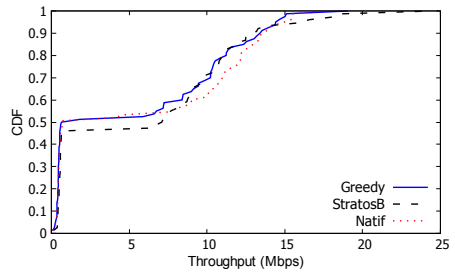

(c) Profile 3

Fig. 5: Throughput (Mbps)

it decreases the latency to $10 \mathrm{~ms}$ compared to $70 \mathrm{~ms}$ at $90^{\text {th }}$ percentile in Greedy.

In Fig. 4a, StratosB and Greedy do not capture that the high packet rate flows (even if it is incurring low bandwidth) can degrade the I/O-bound VNFs performance, like the FW and the NAT in the studied network chains. In Fig. 4b, we apply a high bandwidth traffic. StratosB properly recognises the needed VNF instances but it partially succeeds in making a correct traffic distribution since it does not consider the packet rate when dealing with flows traversing an I/O-bound VNF. For P3, StratosB still performs well with high packet rate flows but not better than Natif since there is still high packet rate flows that need to be suitably distributed, thus, the results in Fig. 4b. Greedy focuses on the horizontal scaling of the VNFs, which does not help when the VNF has a single-threaded implementation so it will not be able to use all the allocated cores. Fig. 5a and $5 \mathrm{~b}$ pick out a slightly identical behaviour of the throughput (Mbps) in the three approaches. However, Fig. $5 \mathrm{c}$ shows how Natif achieves $8 \%$ better throughput compared to Greedy and StratosB.

To conclude, Natif has mitigated the end-to-end delay without sacrificing the network throughput (Mbps), and in some cases, it improves both metrics simultaneously (P3).

\section{Traffic distribution and Arima model evaluation}

In this section, we measure the runtime of the traffic distribution module (also called flow mapping) of the three studied approaches, the CPU utilisation at the controller, and the prediction model efficiency. We define three data-sets that are the working data for the flow mapping. DS1: consisting of $100 \mathrm{VNFs}, 30$ chains, and 1k flows. DS2: $200 \mathrm{VNFs}, 60$ chains, and 2k flows. DS3: $300 \mathrm{VNFs}, 90$ chains, and 3k flows.

In Fig. 6a, Natif outperforms StratosB, and Greedy has the shortest runtime. The runtime evolves linearly with larger data-sets, e.g., in Natif, it increases from 1s to $2.3 \mathrm{~s}$ to $3.3 \mathrm{~s}$ and in StratosB from 1.3s to 2.8s to 3.8s, for DS1, DS2, and
DS3, respectively. To explain this, we look back at how these approaches work. For example, Greedy distributes traffic on a static chain so it is taking the shortest runtime. When dealing with CPU-bound VNF, Natif and StratosB have almost a similar behaviour. Whereas, when it comes to traffic traversing an I/O-bound VNF, StratosB still considers the bandwidth criterion while Natif primarily looks at the packet rates and then the traffic bandwidth, in low priority. As a result, for the case of I/O-bound VNF, when considering the bandwidth rather than the packet rate, we can have more VNF instances since an I/O-bound VNF has less sensitivity to the bandwidth (e.g., NAT) than a CPU-bound VNF. Because spinning out more instances in StratosB, it is taking more time than Natif.

Fig. $6 \mathrm{~b}$ shows the effect of the different algorithms on the CPU usage of the controller node. In DS1 and DS2, Stratos $B$ have the most significant CPU usage, then comes Natif, and lastly Greedy. As highlighted above, this reflects the logic behind each approach. However, in DS3, Natif slightly demands more computational resources than StratosB. Natif runs more iterations than the other approaches, and this may have a clearer repercussion especially with larger data-sets

Lastly, we have conducted a separate experiment consisting of predicting future traffic attributes (bandwidth and packet rate) based on past data of real traffic traversing a VNF (NAT). First, we trained Arima model with data of past 900 seconds (15 min) and then we measured the prediction accuracy. As shown in Fig. 6c, Arima model performs well in predicting the RTT of the ingress flows with an error of less than 30 packets per second.

\section{RELATED WORK}

VNF-VITAL [11] has presented a framework for VNF characterisation. The study has been based on Clearwater IMS VNF and two IDS VNFs (Snort and Suricata). It examines the horizontal and vertical scaling impact on the VNF performance regarding the CPU and memory utilisation. However, the 


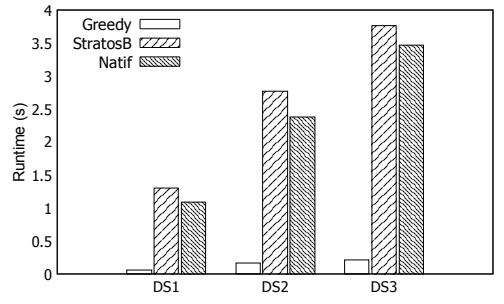

(a) Flow mapping module

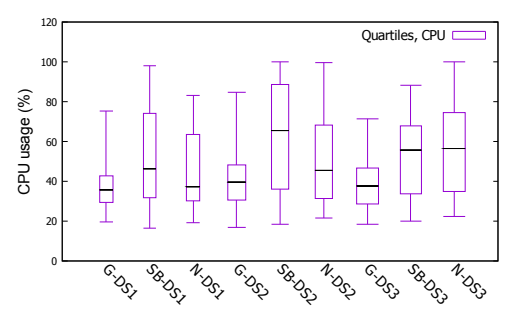

(b) CPU usage at the controller node

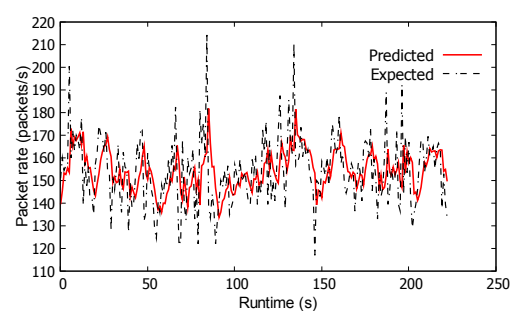

(c) Prediction model evaluation

Fig. 6: Evaluation of flow mapping module, controller node CPU usage, and prediction model. G, SB, and N refer to Greedy, StratosB, and Natif, respectively

study shows no consideration of the possible performance interference incurred by the VNFs since the evaluation did not cover performance of sequence of VNFs where chained, which we have achieved in this paper.

The authors in [2] have focused on an interesting aspect in the service chains. They have illustrated the traffic changing effects of middleboxes and formulated the middleboxes placement problem to minimise the maximum link load ratio. Their proposed VNF chaining is relying on the gain/drop factor of each VNF but neglecting the fact that such chaining should fulfill well defined requirements such as the order of the VNF within the chain. Otherwise, this could probably lead to breaching the network policies that should have been correctly implemented by the chains. Our approach carefully considers the gain/drop factor without attempting any re-ordering that can violate policies.

Work presented in [12] has proposed a graph neural network-based algorithm that predicts future VNF components (VNFCs) resource requirements based on the collected CPU and RAM utilisation data. So, it would be possible to proactively allocate necessary resources to the VNFCs even before they could experience performance degradation. However, we believe that in some cases only considering the CPU or memory utilisation to understand the VNF performance would not be sufficient since the CPU usage metric can be misleading such as in the case of pfSense NAT. In our work, we have shown how the high CPU usage at pfSense NAT does not reflect the real computation need but it is a consequence of high CPU interrupts caused by the high rate of incoming packets (only $2 \%$ effective computation of the total CPU usage).

Other approaches such as [4][5][7] focus on the placement problem to minimise the end-to-end delay at the chains without considering the VNF performance. Instead, they model and manage the VNFs as identical entities.

\section{CONCLUSion AND Future Work}

NFV has facilitated the deployment and management of VNFs, but it has deepened the virtualisation overhead which particularly hurts the performance of latency-sensitive applications. To compensate this overhead, we have proposed a simple but efficient solution that leverages the knowledge on VNFs to propose a VNF qualitative categorisation and chain composition proven useful in minimising the end-to-end delay.
However, apart from the simplified mathematical model, some issues remain unaddressed. Therefore, we aim in future work to answers to following questions. How to find out the gain/drop factor of certain VNFs such as Redundancy Eliminator since, in this case, the factor primarily depends on the packet payload (unlike NAT or proxy where the factor is known and static). Also, how we can tweak our approach to be applicable for bursty traffic where the Arima prediction algorithm would have insufficient time space to be trained.

\section{REFERENCES}

[1] A. Gember, R. Grandl, A. Anand, T. Benson, and A. Akella, "Stratos: Virtual middleboxes as first-class entities," UW-Madison TR1771, p. 15, 2012.

[2] W. Ma, J. Beltran, Z. Pan, D. Pan, and N. Pissinou, "Sdn-based traffic aware placement of nfv middleboxes," IEEE Transactions on Network and Service Management, vol. 14, no. 3, pp. 528-542, 2017.

[3] J. Zhang, F. Ren, and C. Lin, "Survey on transport control in data center networks," IEEE Network, vol. 27, no. 4, pp. 22-26, 2013.

[4] A. Hirwe and K. Kataoka, "Lightchain: A lightweight optimisation of vnf placement for service chaining in nfv," in 2016 IEEE NetSoft Conference and Workshops (NetSoft). IEEE, 2016, pp. 33-37.

[5] S. Sahhaf, W. Tavernier, D. Colle, and M. Pickavet, "Network service chaining with efficient network function mapping based on service decompositions," in Network Softwarization (NetSoft), 2015 1st IEEE Conference on. IEEE, 2015, pp. 1-5.

[6] L. Cui, F. P. Tso, D. P. Pezaros, W. Jia, and W. Zhao, "Plan: Joint policy- and network-aware vm management for cloud data centers," IEEE Transactions on Parallel and Distributed Systems, vol. 28, no. 4, pp. 1163-1175, April 2017.

[7] Y. Zhang, B. Anwer, V. Gopalakrishnan, B. Han, J. Reich, A. Shaikh, and Z.-L. Zhang, "Parabox: Exploiting parallelism for virtual network functions in service chaining," in Proceedings of the Symposium on SDN Research. ACM, 2017, pp. 143-149.

[8] C. Guo, L. Yuan, D. Xiang, Y. Dang, R. Huang, D. Maltz, Z. Liu, V. Wang, B. Pang, H. Chen et al., "Pingmesh: A large-scale system for data center network latency measurement and analysis," $A C M$ SIGCOMM Computer Communication Review, vol. 45, no. 4, pp. 139$152,2015$.

[9] H. Kellerer, U. Pferschy, and D. Pisinger, "Other knapsack problems," in Knapsack Problems. Springer, 2004, pp. 389-424.

[10] H. Z. Moayedi and M. Masnadi-Shirazi, "Arima model for network traffic prediction and anomaly detection," in Information Technology, 2008. ITSim 2008. International Symposium on, vol. 4. IEEE, 2008, pp. $1-6$.

[11] L. Cao, P. Sharma, S. Fahmy, and V. Saxena, "Nfv-vital: A framework for characterizing the performance of virtual network functions," in Network Function Virtualization and Software Defined Network (NFVSDN), 2015 IEEE Conference on. IEEE, 2015, pp. 93-99.

[12] R. Mijumbi, S. Hasija, S. Davy, A. Davy, B. Jennings, and R. Boutaba, "Topology-aware prediction of virtual network function resource requirements," IEEE Transactions on Network and Service Management, vol. 14, no. 1, pp. 106-120, 2017. 